Article

\title{
Knockdown of the Trehalose-6-Phosphate Synthase Gene Using RNA Interference Inhibits Synthesis of Trehalose and Increases Lethality Rate in Asian Citrus Psyllid, Diaphorina citri (Hemiptera: Psyllidae)
}

\author{
Xinyu Liu ${ }^{\dagger}$, Zhiwen Zou ${ }^{\dagger}$, Cong Zhang, Xian Liu, Jing Wang, Tianrong Xin and Bin Xia *(1) \\ School of Life Sciences, Nanchang University, Nanchang 330031, China; ncuskliuxinyu@163.com (X.L.); \\ zouzhiwen@ncu.edu.cn (Z.Z.); 352428819012@email.ncu.edu.cn (C.Z.); ct934@163.com (X.L.); \\ wangjingyn@126.com (J.W.); xintianrong@ncu.edu.cn (T.X.) \\ * Correspondence: xiabin9@163.com; Tel.: +86-13617915100 \\ + These authors contributed equally to this paper.
}

Received: 16 August 2020; Accepted: 4 September 2020; Published: 6 September 2020

check for updates

Simple Summary: In this study, we cloned and characterized a trehalose-6-phosphate synthase (TPS) gene from D. citri (DcTPS) for the first time. Meanwhile, we used RNA interference (RNAi) technology to efficiently disrupt DCTPS gene function in order to elucidate its role in the growth and development of $D$. citri. Our results suggest that dsRNA-mediated gene-specific silencing resulted in a strong reduction in relative expression of DcTPS and survival rate of nymphs, as well as an increase in malformation. This work was undertaken to establish a foundation for further research on the functions of $D$. citri trehalose-6-phosphate synthase. This will provide a new target for the control of D. citri in the field.

\begin{abstract}
Diaphorina citri Kuwayama is the vector of citrus "huanglongbing", a citrus disease which poses a significant threat to the global citrus industry. Trehalose-6-phosphate synthase (TPS) plays an important role in the regulation of trehalose levels of insects, while its functions in D. citri are unclear. In this study, full-length cDNA sequences of the TPS gene from D. citri (DcTPS) were cloned and its expression patterns at various developmental stages were investigated. The results indicated that DcTPS mRNA was expressed at each developmental stage and the highest DcTPS expression was found in the fifth-instar nymphs of $D$. citri. Additionally, mortality and deformity of $D$. citri were observed after 24 and $48 \mathrm{~h}$ by feeding with three different dsRNA concentrations $(20,100$ and $500 \mathrm{ng} / \mu \mathrm{L})$. The results indicated that DcTPS expression was declined, and mortality and malformation in nymphs were increased via feeding with dsDcTPS. Moreover, the enzyme and trehalose content were decreased, while the content of glucose was significantly higher than that of untreated (control) individuals. This suggests that DcTPS might be vital for the growth and development of $D$. citri and further studies of the genes should be related to molting and metabolism for controlling D. citri.
\end{abstract}

Keywords: Diaphorina citri; trehalose-6-phosphate synthase; feeding; RNA interference; lethal phenotypes

\section{Introduction}

The Asian citrus psyllid, Diaphorina citri Kuwayama (Hemiptera: Psyllidae), is a notorious pest that causes huge economic loss to the citrus industry all around the world [1-3]. The psyllids can excrete copious amounts of honeydew, leading to bituminous coal sickness [4]. In addition, D. citri 
serves as the only vector of the bacterium Candidatus Liberbacter asiaticus, which is responsible for outbreaks of "huanglongbing (HLB)", and seriously affects economic development in the citrus industry [2,5-7]. Therefore, the control of $D$. citri plays a crucial role in preventing HLB from spreading [8]. Previous studies in China have shown that reduction of $D$. citri populations depended on the widespread application of insecticides $[2,9,10]$. Nowadays, control of $D$. citri still mainly depends on the widespread use of insecticides [11]. However, excessive use of insecticides can result in detrimental effects on human health, including poisoning, and contamination of the environment $[12,13]$. Therefore, exploring new and effective means of control of $D$. citri populations is urgent for sustainable economic development of the citrus industry.

RNA interference (RNAi) is a method for posttranscriptional gene silencing that has become a popular tool for exploring the function of genes in various insects [8]. Studies have shown that methods of transporting double-stranded RNA (dsRNA) into organisms mainly include microinjection, soaking, oral feeding and transgenic plant expression [14-16]. These methods were successfully applied to silencing of specific genes in various insects as potential pest control targets [17-19]. Previous research has shown that the expression of six major genes involved in the chitin biosynthesis pathway and one main gene involved in lipid catabolism was suppressed after injection with dsRNA of trehalose-6-phosphate synthase in Heortia vitessoides (HvTPS) [20]. Previous studies demonstrated that Nilaparvata lugens nymphs fed with dsTPS experienced a significant reduction in the expression of the target gene and survival rate [21]. Additionally, silencing the TPS gene of Tribolium castaneum resulted in malformation and an increased mortality rate; chitin metabolism of T. castaneum was also affected [22]. The efficacy of different dsRNA delivery methods varies with the target genes [8]. Microinjection is considered the most efficient means of knocking down target genes [23,24]. However, RNAi delivery by microinjection is difficult to achieve in small insects such as the nymphs of $D$. citri because the injection procedure can cause injury and result in abnormal death. Overall, oral feeding is considered an excellent method of gene silencing in the brown planthopper, Nilaparvata lugens [21]. Therefore, we hypothesized that oral feeding could be useful for silencing a gene of the nymphs of D. citri due to its expediency.

Trehalose, a non-reducing disaccharide formed by two glucose molecules linked by a $1 \alpha-1 \alpha$ bond, exists in various organisms, including plants, algae, fungus, yeasts, bacteria, insects and so on [25-28]. However, trehalose is not found in mammals [28]. Trehalose is also called "blood sugar" due to its important physiological functions in insects $[29,30]$. Trehalose is synthesized by trehalose-6-phosphate synthase (TPS) and trehalose-6-phosphate phosphatase (TPP) [31]. The TPS gene in insects was first reported in Drosophila melanogaster [32]. To date, many TPS genes have been reported from several insects, including Heortia vitessoides (Lepidoptera: Crambidae) [20], Locusta migratoria manilensis (Orthoptera: Acrididae) [33], Tribolium castaneum (Coleoptera: Tenebrionidae) [22], Spodoptera exigua (Lepidoptera: Noctuidae) [34], the brown planthopper, Nilaparvata lugens Stål (Hemiptera: Delphacidae) [24] and Catantops pinguis (Orthoptera: Catantopidae) [35]. However, the TPS mode of functionality has not been reported in D. citri.

In this study, DcTPS, a TPS gene from D. citri, was cloned and characterized for the first time and its expression patterns at each developmental stage were investigated by reverse transcription quantitative polymerase chain reaction (RT-qPCR). Furthermore, RNA interference (RNAi) technology was used to disrupt DcTPS gene function in order to elucidate its role in the development of $D$. citri, including phenotypic changes, mortality, the expression level of DcTPS gene, the content of trehalose and glucose and the content of trehalose-6-phosphate synthase. These findings will be conducive to further study of the role of DCTPS in metamorphosis and carbohydrate metabolism. Furthermore, RNAi of the DCTPS gene is expected to provide a technical platform and theoretical reference as a potential biological pesticide for efficient and sustainable control of $D$. citri in the future. 


\section{Materials and Methods}

\subsection{D. citri Rearing and Sample Collection}

In this study, healthy adults of D. citri were collected from Murraya exotica plants in Donghu Park, Quanzhou city, Fujian Province. Then, D. citri was maintained in our laboratory for over 3 years and reared continuously in Murraya exotica in insect rearing cages $\left(60 \times 60 \times 90 \mathrm{~cm}^{3}\right)$. In the meantime, it was not disturbed by any insecticide. The temperature-controlled growth rooms, maintained at the laboratory building of School of Life Science in Nanchang University, were set at $27 \pm 1{ }^{\circ} \mathrm{C}$ and $\mathrm{RH}$ (relative humidity) $70 \pm 5 \%$, with a photoperiod of 14:10 (L:D).

Following the methodology of Yu et al (2020), 100 post-mating D. citri females were collected and released onto fresh Murraya exotica plants placed in an insect rearing cage for obtaining $D$. citri individuals of the same growth and development stage. D. citri nymphs were classified into different stages based on their morphological features, and we continuously collected the nymphs by using a brush until the adults appeared [36]. Seven stages of D. citri were used for analysis of the different developmental stages' expression levels of DcTPS gene including egg, first-, second-, third-, fourth-, fifth-instar nymphs and adults. Thirty individuals of D. citri were used for each sample collection. All stages were performed with three replicates.

\subsection{RNA Isolation and DcTPS $c D N A$ Synthesis}

Firstly, total RNA was extracted from each sample using the Eastep ${ }^{\circledR}$ Super total RNA Extraction Kit (Shanghai Promega Trading Co., Ltd., Shanghai, China). Each tube of collected sample was homogenized in an ice bath with $300 \mu \mathrm{L}$ of lysis solution. Then, $300 \mu \mathrm{L}$ RNA diluent was added into the tube and mixed well. The sample was heated at $70{ }^{\circ} \mathrm{C}$ for $5 \mathrm{~min}$ and centrifuged at $4{ }^{\circ} \mathrm{C}, 14,000 \times g$, for $5 \mathrm{~min}$. Then, $500 \mu \mathrm{L}$ of supernatant was transferred to another new tube, and $250 \mu \mathrm{L}$ of absolute ethyl alcohol was added and mixed well. The mixture was transferred to a new centrifugal column installed on a collecting pipe and centrifuged at $4{ }^{\circ} \mathrm{C}, 14,000 \times g$, for $1 \mathrm{~min}$. At the same time, the filtrate was discarded, and $600 \mu \mathrm{L}$ RNA lotion was added and centrifuged at $4{ }^{\circ} \mathrm{C}, 14,000 \times g$, for $1 \mathrm{~min}$. Fifty $\mu \mathrm{L}$ of the prepared DNase 1 incubation solution was added to the adsorption film center and incubated at room temperature for $15 \mathrm{~min}$. Then, $600 \mu \mathrm{L}$ RNA lotion was added and centrifuged at $4{ }^{\circ} \mathrm{C}, 14,000 \times g$, for $45 \mathrm{~s}$, repeating the process twice. Meanwhile, the filtrate was discarded. The centrifugal column was installed on the collecting pipe and centrifuged at $4{ }^{\circ} \mathrm{C}, 14,000 \times g$, for $2 \mathrm{~min}$. Next, the centrifugal column was anew installed on an elution tube and $100 \mu \mathrm{L}$ nuclease-free water was added. Solutions were kept at room temperature for $2 \mathrm{~min}$ and centrifuged at $4{ }^{\circ} \mathrm{C}, 14,000 \times g$, for $1 \mathrm{~min}$. The RNAs were stored at $-80^{\circ} \mathrm{C}$. Simultaneously, the concentration and purity of RNA were assayed by NanoPhotometer N60 Touch (IMPLEN GMBH, Munich, Germany) at absorbance ratios of A260/230 and A260/280. The integrity of the total RNA was verified via 1\% agarose gel electrophoresis. In accordance with the manufacturer's instructions, total RNA was reverse-transcribed using the PrimeScript ${ }^{\mathrm{TM}}$ II 1st Strand cDNA Synthesis Kit (Takara Biomedical Technology (Beijing) Co., Ltd., Beijing, China). In other words, $1.0 \mu \mathrm{L}$ of random 6 mers, $1.0 \mu \mathrm{L}$ of dNTP mixture and $8 \mu \mathrm{L}$ of total RNA were mixed to reach $10 \mu \mathrm{L}$ in the tube, which was then incubated at $65{ }^{\circ} \mathrm{C}$ for 5 min to improve reverse transcription efficiency. Then, $4.0 \mu \mathrm{L}$ of $5 \times$ PrimeScript II Buffer, $0.5 \mu \mathrm{L}$ of RNase Inhibitor and $1.0 \mu \mathrm{L}$ Primer Script II RTase and RNase-free water was added to reach $20 \mu \mathrm{L}$. Finally, the mixture was incubated at $45^{\circ} \mathrm{C}$ for $50 \mathrm{~min}$ and then incubated at $70{ }^{\circ} \mathrm{C}$ for $15 \mathrm{~min}$. The cDNA was stored at $-20^{\circ} \mathrm{C}$ for subsequent experiments.

\subsection{Molecular Cloning}

Fragments of the putative DcTPS gene were procured from the transcriptome database for D. citri. The veracity of the sequences was established by polymerase chain reaction (PCR) using the primers in Table 1. Full-length cDNA was obtained by 5'- and 3'-RACE using SMARTer ${ }^{\circledR}$ RACE5'/3' kit (Takara Biomedical Technology (Beijing) Co., Ltd., Beijing, China) with the specific primers listed in Table 1. 
We subsequently recovered and purified the PCR product. The purified DNA was ligated onto the PGEM-Teasy Vector (Shanghai Promega Trading Co., Ltd., Shanghai, China) and the dideoxynucleotide method was used for sequencing (Sangon Biotech, Shanghai, China).

Table 1. Primers used in this study.

\begin{tabular}{|c|c|c|c|}
\hline Primer Names & Primer Sequences & Length of Primer & Primer Usage \\
\hline DcTPS-F & ATGCTTGCCGCCAACACT & $18 \mathrm{bp}$ & \multirow{2}{*}{ Middle fragment } \\
\hline DcTPS-R & CGCATCCCGATAGAACGA & $18 \mathrm{bp}$ & \\
\hline Dc5'TPS-R1 & CCTCCAATGTTCGGCACAAA & $20 \mathrm{bp}$ & \multirow{2}{*}{ 5'RACE } \\
\hline Dc5'TPS-R2 & CCAGAAGGTGCCATTACAGC & $20 \mathrm{bp}$ & \\
\hline Dc3'TPS-F1 & GCTCTAACAATGCCCGAGGAC & $21 \mathrm{bp}$ & \multirow{2}{*}{ 3'RACE } \\
\hline Dc3'TPS-F2 & ACAATGCCCGAGGACGAG & $18 \mathrm{bp}$ & \\
\hline UPM long & CTAATACGACTCACTATAGGGCAAGCAGTGGTATCAACGCAGAGT & $45 \mathrm{bp}$ & \\
\hline UPM short & CTAATACGACTCACTATAGGGC & $22 \mathrm{bp}$ & \\
\hline NUP & AAGCAGTGGTAACAACGCAGAGT & $23 \mathrm{bp}$ & \\
\hline B-Actin-F & CCCTGGACTTTGAACAGGAA & $20 \mathrm{bp}$ & \multirow{6}{*}{ RT-qPCR } \\
\hline B-Actin-R & CTCGTGGATACCGCAAGATT & $20 \mathrm{bp}$ & \\
\hline$\alpha$-tubulin-F & GGTTCAAGGTGGGTATCAACTAT & $23 \mathrm{bp}$ & \\
\hline$\alpha$-tubulin-R & TAGCGGTGGTGTTGGAAAG & $19 \mathrm{bp}$ & \\
\hline Q-DcTPS -F & AGGGAATGCTAGGTTGTGAT & $20 \mathrm{bp}$ & \\
\hline Q-DcTPS -R & TGCTCTACCAGGAGGTTCTT & $20 \mathrm{bp}$ & \\
\hline dsGFP-F & TAATACGACTCACTATAGGGAAGGGCGAGGAGCTGTTCACCG & $42 \mathrm{bp}$ & \multirow{4}{*}{ dsRNA synthesis } \\
\hline dsGFP-R & TAATACGACTCACTATAGGGCAGCAGGACCATGTGATCGCGC & $42 \mathrm{bp}$ & \\
\hline dsDcTPS-F & TAATACGACTCACTATAGGGTCGTCGACTGTTGTCAGAGG & $40 \mathrm{bp}$ & \\
\hline dsDcTPS-R & TAATACGACTCACTATAGGGAGAACGACGCCAGTTCATCT & $40 \mathrm{bp}$ & \\
\hline
\end{tabular}

\subsection{Bioinformatic and Phylogenetic Analyses}

The cDNA sequence of DcTPS was translated with the Translate tool (http://www.expasy.org/ translate/). Amino acid sequences were deduced using ExPASy (http://web.expasy.org/translate). The molecular weight (MW) and isoelectric point (pI) of the deduced amino acid sequences were predicted by Compute pI/Mw (http://web.expasy.org/compute_pi/). The N-linked glycosylation sites were analyzed using NetNGlyc 1.0 Server (http://www.cbs.dtu.dk/services/NetNGlyc/). Sequence comparisons were performed using DNAMAN. Additionally, a phylogenetic tree was constructed using a total of 20 insect TPS protein sequences obtained from NCBI via MEGA7.0 software and Clustal $X 1.83$ by the maximum likelihood method. Bootstrap values were calculated based on 1000 replicates [37].

\subsection{Expression of DcTPS Gene}

The cDNA templates derived from different developmental stages of $D$. citri were used for temporal expression tests. Primers were designed for quantitative real time (RT-qPCR) by Prime 5.0 and are listed in Table 1. Expression of the target gene was measured by RT-qPCR and normalized with two stable reference genes, $\beta$-Actin (GenBank: DQ675553) and $\alpha$-tubulin gene (GenBank: DQ675550) [3]. Each PCR reaction was mixed with $10 \mu \mathrm{L}$ TB green, $7.8 \mu \mathrm{L}$ ddH $_{2} \mathrm{O}, 1.0 \mu \mathrm{L}$ cDNA, $0.4 \mu \mathrm{L}$ Rox dye and $0.4 \mu \mathrm{L}$ of each primer. The thermal cycling profile consisted of an initial denaturation at $95^{\circ} \mathrm{C}$ for $5 \mathrm{~min}$ and 40 cycles at $95^{\circ} \mathrm{C}$ for $10 \mathrm{~s}$ and $60^{\circ} \mathrm{C}$ for $20 \mathrm{~s}$. The reactions were performed with the StepOnePlus ${ }^{\mathrm{TM}}$ Real-Time PCR Instrument (Thermo Fisher Scientific, Singapore). The relative expression level was calculated using the $2^{-\Delta \Delta \mathrm{Ct}}$ method [38].

\subsection{Preparation of dsRNA and Feeding}

Firstly, dsRNA fragments targeting DcTPS (447 bp) and GFP (GenBank: LN515608) (415 bp) were prepared by using TranscriptAid T7 High Yield Transcription (Thermo Scientific, Lithuania). Secondly, the dsRNA was purified in accordance with instructions for the use of the GeneJET RNA Purification Kit (Thermo scientific, Lithuania). Thirdly, based on the results of a previous study, some adjustments 
were made to our rearing procedure in the dsRNA ingestion experiment [11]. Briefly, glass cylinders $(12.0 \mathrm{~cm}$ in length and $3.0 \mathrm{~cm}$ in diameter) were used as a feeding chamber. The dsRNA was delivered by an artificial diet placed between two layers of stretched Parafilm. The artificial diet consisted of $20 \%$ (w:v) sucrose mixed with dsRNA DcTPS at a final concentration of 20, 100 and $500 \mathrm{ng} / \mu \mathrm{L}$. Meanwhile, dsGFP as a control group was fed in the same way. Thirty newly emerged fifth-instar nymphs of $D$. citri were used for each treatment. Meanwhile, there were three biological replicates in each treatment. The number that survived and molted were calculated 24 and $48 \mathrm{~h}$ after dsRNA feeding. At the same time, individuals that were clearly of abnormal phenotype were photographed using a Leica Microsystems Ltd. (Leica, Singapore) digital camera. Subsequently, the D. citri individuals (at least 10 individuals) surviving different concentrations of dsRNA treatment at $24 \mathrm{~h}$ and $48 \mathrm{~h}$ were kept at $-80{ }^{\circ} \mathrm{C}$ and used for RNA extraction, analysis of relative expression levels and assays of enzyme activity, trehalose content and glucose content. The sample collections of each concentration and each duration were performed with three replicates.

\subsection{Quantitative Detection of Trehalose-6-Phosphate Synthase Content in D. citri}

The content of trehalose-6-phosphate synthase in D. citri was measured using a modified protocol based on a previous report [20]. The content of DcTPS in D. citri was quantified by the insect trehalose-6-phosphate synthase ELISA Assay Kit (Jonln, Shanghai, China). Briefly, each sample containing three surviving individuals of $D$. citri was homogenized in $300 \mu \mathrm{L}$ phosphate buffer saline (PBS, pH 7.0) and centrifuged at $4{ }^{\circ} \mathrm{C}, 5000 \times g$, for $10 \mathrm{~min}$. Then, $100 \mu \mathrm{L}$ of HRP-conjugate reagent was added to $50 \mu \mathrm{L}$ of supernatant and incubated at $37^{\circ} \mathrm{C}$ for $60 \mathrm{~min}$. Liquid was aspirated from each well and all wells were washed with $350 \mu \mathrm{L}$ wash solution, repeating the process four times for a total of five washes. Then, $50 \mu \mathrm{L}$ of chromogen solution $\mathrm{A}$ and $50 \mu \mathrm{L}$ of chromogen solution B were added to each well with gentle mixing and incubated for $15 \mathrm{~min}$ at $37^{\circ} \mathrm{C}$ under protection from light. Then, $50 \mu \mathrm{L}$ of stop solution was added into each well. The absorbance was measured at $450 \mathrm{~nm}$. The content of trehalose-6-phosphate synthase in the sample solution was calculated based on a standard curve. Three technical replicates were required for each sample measurement.

\subsection{Measurements of Trehalose and Glucose Content}

Five surviving individuals of $D$. citri were collected for the trehalose and glucose content assays. Following the assay procedure specified in the Insect Trehalose ELISA Kit and Insect Glucose ELISA Kit (Xinquan, China), samples were homogenized in an ice bath with $100 \mu \mathrm{L}$ of extraction solution and centrifuged at $4{ }^{\circ} \mathrm{C}, 3000 \times g$, for $10 \mathrm{~min}$. Then, $40 \mu \mathrm{L}$ of sample diluent was added to $10 \mu \mathrm{L}$ of testing sample in each well. Then, $100 \mu \mathrm{L}$ of HRP-conjugate reagent was added and the well was incubated at $37^{\circ} \mathrm{C}$ for $1 \mathrm{~h}$. Then, $50 \mu \mathrm{L}$ of chromogen solutions $\mathrm{A}$ and B were mixed in after the wash followed by incubation for $15 \mathrm{~min}$ at $37^{\circ} \mathrm{C}$. The concentrations of trehalose and glucose were measured photometrically at $450 \mathrm{~nm}$. The trehalose and glucose content in the sample solution were calculated based on a standard curve.

\subsection{Statistical Analysis}

The data were summarized as the mean \pm SE (standard error) for all data sets. The data were then subjected to a one-way analysis of variance (ANOVA) using SPSS 26.0. Differences among means were tested using a Student-Newman-Keuls (S-N-K) test for multiple comparisons. All experiments were performed with three biological replicates. Each biological replicate was performed with three technical repetitions. Differences were considered statistically significant at the $5 \%$ level $(p<0.05)$. 


\section{Results}

\subsection{Sequence Analysis of DcTPS cDNA}

The full-length cDNA sequence of DcTPS was cloned and deposited in the GenBank database (MT675285). DcTPS cDNA is made up of 2162 nucleotides with an open reading frame (ORF) of 1785 nucleotides (Figure 1), which encodes a protein of 594 amino acids with a predicted molecular mass of $67.057 \mathrm{kDa}$ and a theoretical isoelectric point $(\mathrm{pI})$ of 4.82. DcTPS has two glycosylation sites. The BLAST analysis revealed that DcTPS shares $76 \%$ similarity identity with other insects' TPS genes. Multiple sequence alignments showed that two signatures (HDYHL and DGMNLV) unique to TPS were well conserved in DcTPS (Figure 2). The phylogenetic tree showed that the DcTPS deduced amino acid sequence was more closely related to TPS from Hemiptera (Acyrthosiphon pisum and Diuraphis noxia) than its counterparts from Diptera, Lepidoptera, Orthoptera and Coleoptera (Figure 3).

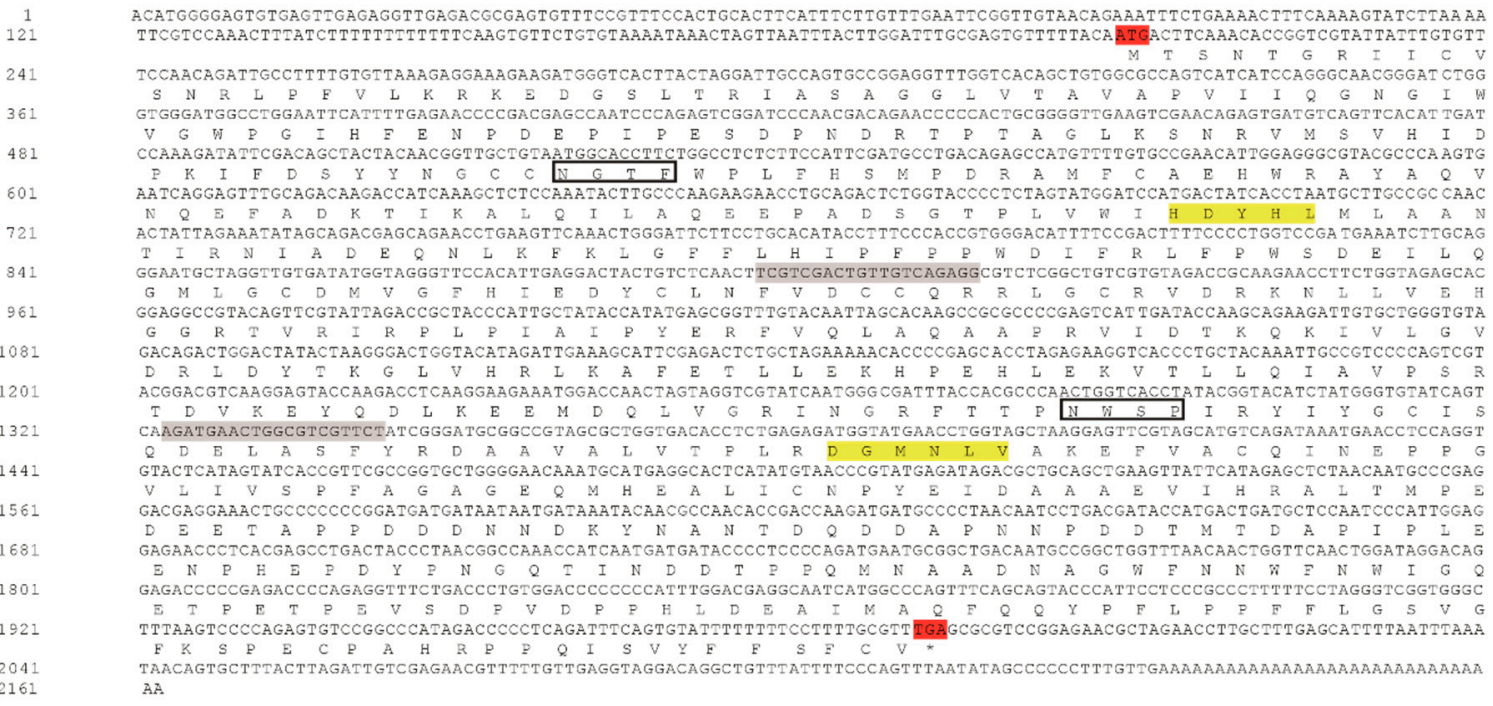

Figure 1. Nucleotide and deduced amino acid sequences of DcTPS from D. citri. Both initiation codon and termination codon are shaded in red. Motifs (or signature motifs) unique to trehalose-6-phosphate synthase (TPS) (residues 450-464 and 555-567) are shaded in yellow. Two glycosylation sites are indicated in black box. The primer sequences used for synthesis of dsRNA are shaded in gray. 

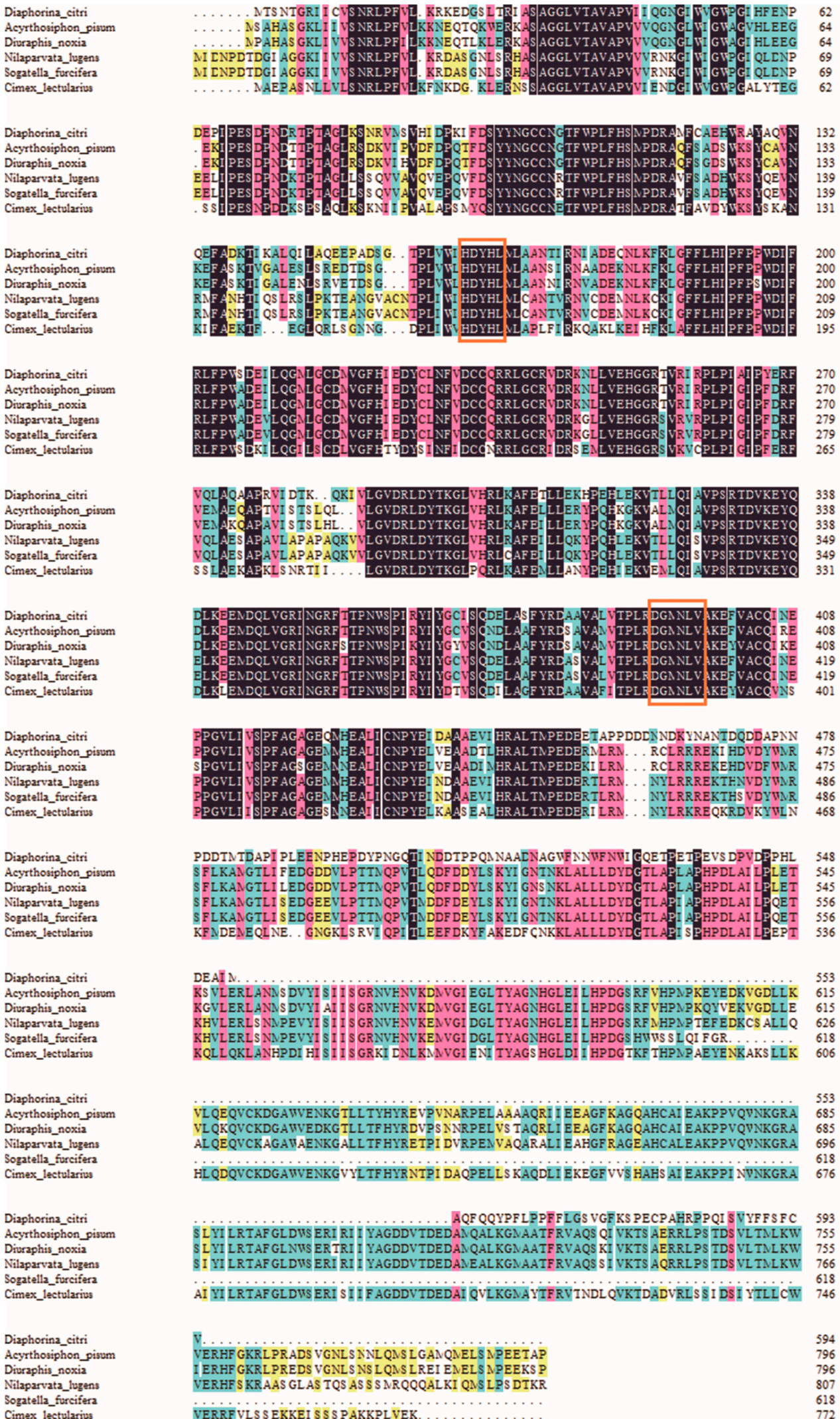

PDDTN TDAPI PLEENPHEPDYPNGCTI NDDTPPQANAALNAGWFNNWFNI GQETPETPEVS DPVDFPHL 548 SFLKANGTLI FEDGDDVL PTTN QPVTLL ODFDDYLS KYI GNTNKL ALLLDYDGTL APL APHPDL AI LPLET 545 SFLKANGTLI LEDGDDVL PTTNQPVIL QDF DDYLS KYI GNS NKL ALLLDYDGTL APL APHPDL AI LPLET 545 SFLKANGTLI S EDGEEVL PTTM QPVITADDF DEYLS KYI GNTNKL AL LLDYDGTL API APHPDL AI LPQET 556

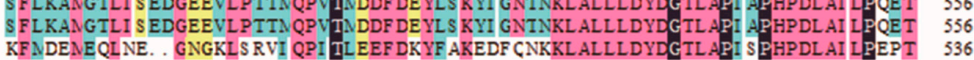

DEAI $N$

553

KS VLERLANNS DVYI I I I SRNVHNVKDNGI EGL TYAGNHGLEI LHPDGS RFVHPNP KE YEDKVGDLLK 615 KGVLERLANNS DVYI AI I S GRNVFNVKDNVGI EGL TYAGNHGLEI LHPDGS RFVHPMPKQYVEKVGDLLE 615 KHVLERL S NMPEVYI II I S GRNVFNVKENVGI DGL TYAGNHGLEI L HPDGS RFMHPNP TEFEDKCS ALLQ 626 KOL LOKL ANHPDI HI $\mathrm{I}$ I I S GRKI DNL KMVGI ENI TYAGS HGLDI I HPDGTKF THPN PAEYENKAKS LLK 606

VLQEQVCKDGAWVENKGTL L TYHYREVPVNARPEL AAAAQRI I EEAGF KAGQAHCAI EAKPPVQVNKGRA 553 VL QKQVCKDG ANVEDKGTL L TF HYRDVPS NNRPEL VS TAQRLI EEAGF KAGQAHCAI EAKPPVQVNKGRA 685 ALQEQVCKAGAVAENKG ALL TF HYRE TPI DVRPENVAQARALI EAHGF RAGEAHCALEAKP PVQVNKGRA 696 HLQDQVCKDGAWVENKGVYL TFHYR NTPI DAQPELL S KAQDLI EKEGFVVS HAHS AI EAKPPI NWNKGRA 676

YYI LRTAFG.......... AQF QQYPFL LPFFLGS VGFKS PECPAHRPPQI SVYFFSFC 593 1 YAGDDVTDEDAN QAL KGNAATFRVAQS QI VKTS AERRL PS TDS VL TMLKW 755 $\begin{array}{ll}\text { SI YI LRTAF GLDWS ERI RI I YAGDDVIDEDANEALKGNAATFRVAQS SI VKTS AQRRLPS TDS VL TMLKW } & 766\end{array}$

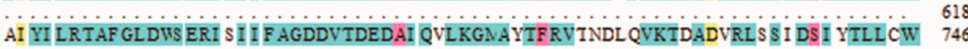

VERHF GKLPRADS VGNLS NNLQNS LGANQMELS MPETAP I ERHF GKRLPREDS VGNLS NS L QMS LREI ENELS NPEEKS P SKRAAS GLAS TQS ASSS MRQQQALKI QASLPS

VERRFVIS S EKKEI S S S PAKKPLVEK.

594
796
796
807
618
772

Figure 2. The amino acid alignment of trehalose-6-phosphate synthase (TPS) sequences from Diaphorina citri (MT675285), Acyrthosiphon pisum (XP_001944221), Diuraphis noxia (XP_015365486), Nilaparvata lugens (ACV20871), Sogatella furcifera (JQ013797), Cimex lectularius (XP_014255923). Signature motifs unique to trehalose-6-phosphate synthase (TPS) (HDYHL and DGMNLV) are indicated with red frame. 


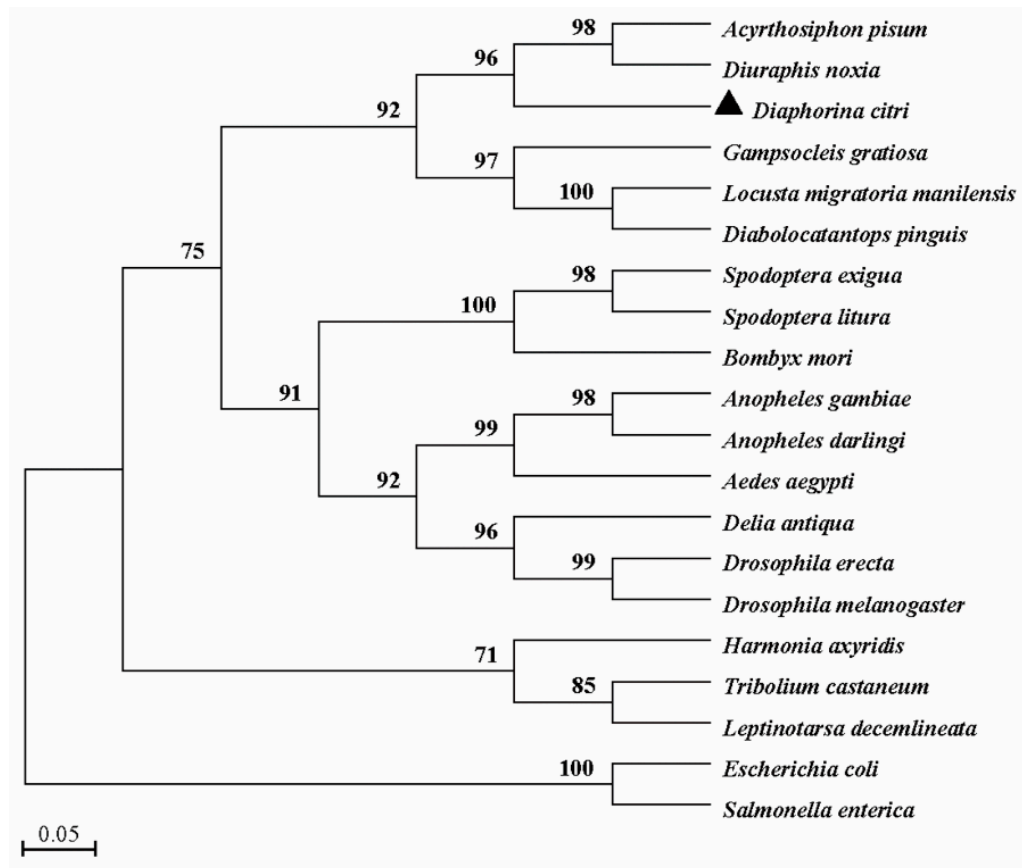

Figure 3. Phylogenetic analysis of DcTPS and TPSs from other species. The phylogenetic tree was constructed based on the amino acid sequences of known TPSs. Full-length amino acid sequences were aligned with the Mega 7.0 program to generate the phylogenetic tree by the maximum likelihood method. A bootstrap analysis was carried out, and the robustness of each cluster was verified with 1000 replicates. Values at the cluster branches indicate the results of the bootstrap analysis. The sequences were obtained from GenBank under the following accession numbers: Acyrthosiphon pisum (XP_001944221), Diuraphis noxia (XP_015365486), Gampsocleis gratiosa (APZ77037), Locusta migratoria manilensis (EU131894), Diabolocatantops pinguis (ACV32626), Spodoptera exigua (ABM66814), Spodoptera litura (ADA63844), Bombyx mori (XP_004926812), Anopheles gambiae (XP_317243), Anopheles darling (ETN66003), Aedes aegypti (XP_001657813), Delia antiqua (AFW99833), Drosophila erecta (XP_001968664), Drosophila melanogaster (NP608827), Harmonia axyridis (FJ501960), Tribolium castaneum (XP_975776), Leptinotarsa decemlineata (AO799586), salmonella enterica (PXW07297) and Escherichia coli (NP416410). Scale bar represents 0.05 substitutions per site.

\subsection{Developmental Stage-Specific Expression Pattern of DcTPS}

The relative expression levels of DcTPS at various stages were determined by RT-qPCR. The results suggested that DcTPS is continuously expressed at all developmental stages $\left(\mathrm{F}_{6,14}=173.482, p=0.0001\right)$. The expression of DcTPS increased steadily from the egg stage and reached a maximum in fifth-instar nymphs (Figure 4). The expression of DcTPS in adults declined slightly (Figure 4). As shown by the data, the expression level of DcTPS in the fifth-instar nymphs was 19.13 times higher than in the eggs. At the same time, the expression level in adult D. citri was 8.93 times higher than in the eggs. The different temporal expression patterns evident from the data suggest distinct physiological roles of DcTPS. 


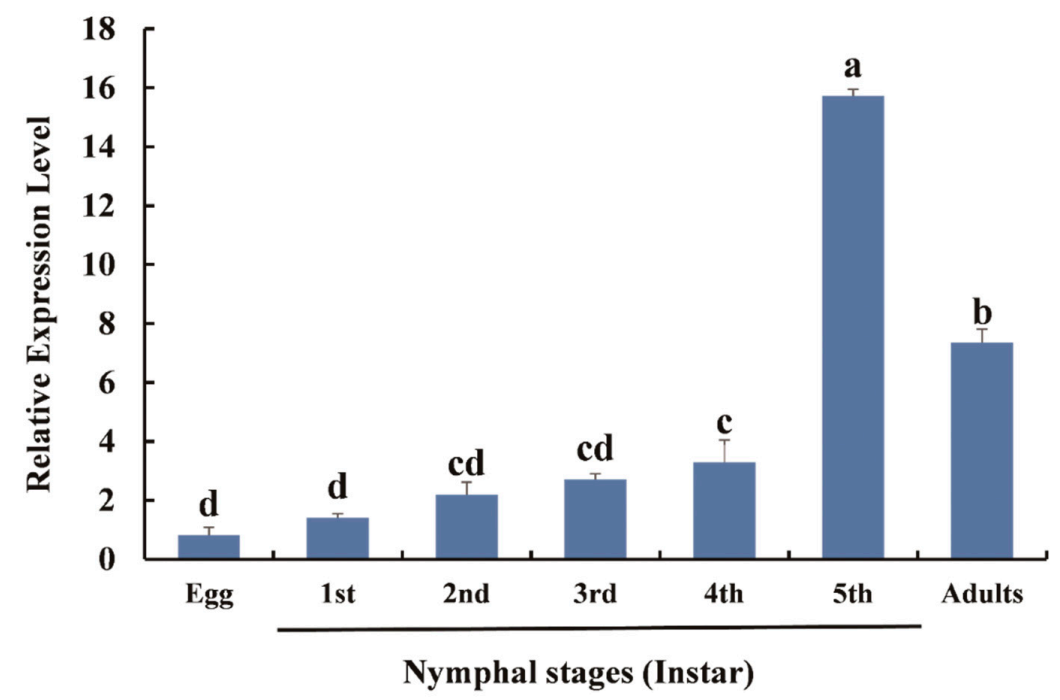

Figure 4. Expression patterns of DcTPS at different developmental stages of D. citri. Relative expression levels of DcTPS were analyzed using RT-qPCR and calculated with the $2^{-\Delta \Delta C t}$ method. SPSS 26.0 software was used for statistical analysis. Bars with difference letters are significantly different according to Student-Newman-Keuls (S-N-K) test $(p<0.05)$.

\subsection{Phenotype and Survival Rate Analysis after Feeding with dsRNA}

With the successful silencing of the DcTPS gene, D. citri subjected to RNAi exhibited abnormal phenotypes after feeding with dsDcTPS (Figure 5B,C). The data on survival rates of nymphs at 24 and $48 \mathrm{~h}$ showed that there were significant differences $(p<0.05)$ between control and 20, 100 and $500 \mathrm{ng} / \mu \mathrm{L}$, with average survival rates of $97,61,51,43 \%$, respectively (Figure $6 \mathrm{~A}$ ). The decreased survival probability in D. citri at $24 \mathrm{~h}\left(\mathrm{~F}_{3,8}=105.333, p=0.0001\right)$ and $48 \mathrm{~h}\left(\mathrm{~F}_{3,8}=208.889, p=0.0001\right)$ can be attributed to the applied doses of dsDcTPS (Figure 6A).
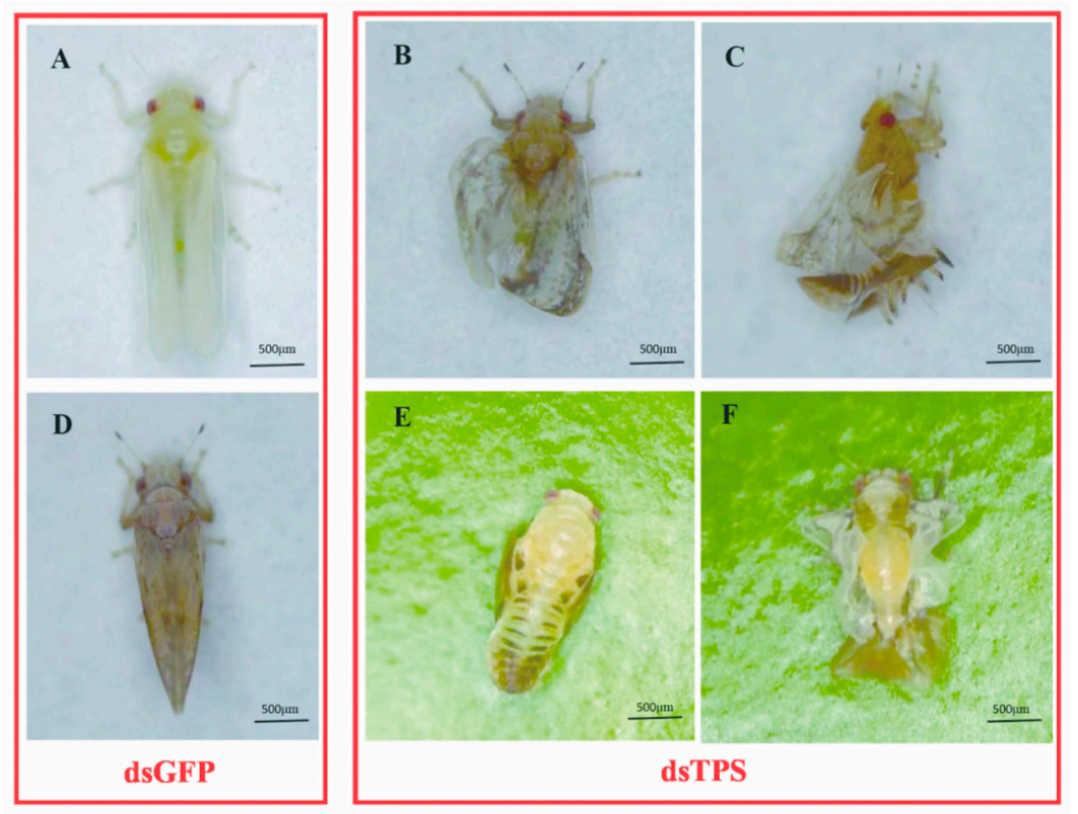

Figure 5. Effects of RNAi on D. citri representative phenotypes after feeding with dsRNA. (A,D) A newly emerged adult and mature adult come from the fifth-instar nymph treated with dsGFP (control). $(\mathbf{B}, \mathbf{C}, \mathbf{E}, \mathbf{F})$ D. citri that underwent metamorphosis after being treated with dsDcTPS. 

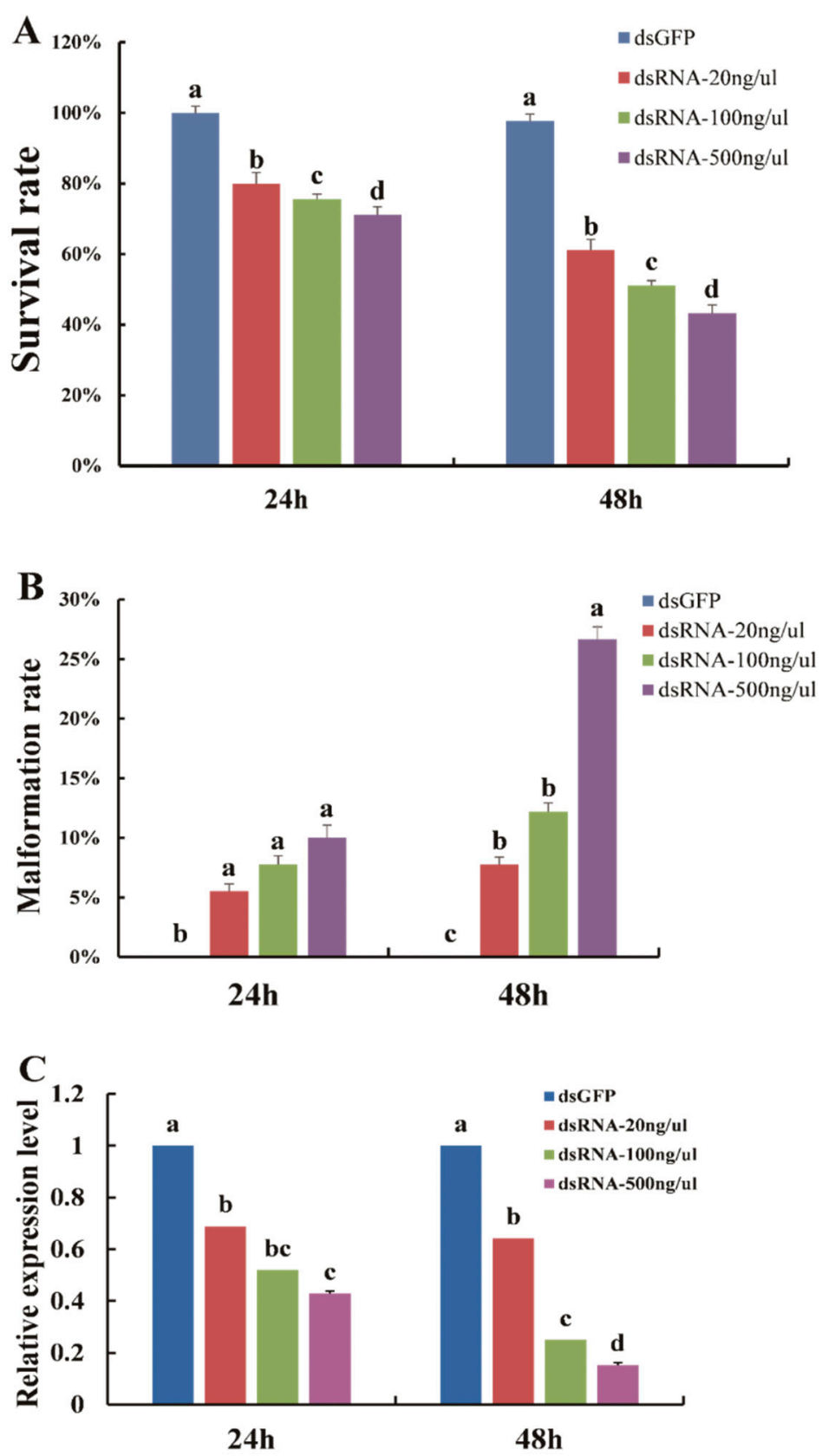

Figure 6. Effects of feeding dsDcTPS on survival rate (A), malformation rate (B) and relative expression levels (C) in D. citri. Results of control (dsGFP) and treatment groups (dsDcTPS of 20, 100 and $500 \mathrm{ng} / \mu \mathrm{L}$ ) 24 and $48 \mathrm{~h}$ post RNAi are shown. Statistical analysis was conducted using SPSS 26.0 software. Bars with different letters are significantly different in terms of SNK test $(p<0.05)$.

Furthermore, after continuous feeding on the dsDcTPS-containing diet, the average malformation rate increased to 7,12 and $26 \%$ on treatments after $48 \mathrm{~h}\left(\mathrm{~F}_{3,8}=36.970, p=0.0001\right)$ separately. This was significantly higher than malformation rates in the dsGFP group (Figure $6 \mathrm{~B}$ ).

To demonstrate whether target mRNA was suppressed though feeding on the dsDcTPS diet, the relative expression levels of the DcTPS gene were compared between the dsGFP and dsDcTPS (20, 100 , and $500 \mathrm{ng} / \mu \mathrm{L})$ treatments at $24 \mathrm{~h}\left(\mathrm{~F}_{3,8}=343.308, p=0.0001\right)$ and $48 \mathrm{~h}\left(\mathrm{~F}_{3,8}=24.442, p=0.0001\right)$. The results indicated that the relative expression level of DcTPS in D. citri decreased with increasing rates of applied dsDcTPS (Figure $6 \mathrm{C})$. The most serious inhibition level $(\sim 85 \%)$ was observed at $500 \mathrm{ng} / \mu \mathrm{L}$ after $48 \mathrm{~h}$ (Figure $6 \mathrm{C}$ ). 
Furthermore, compared to the dsGFP group (Figure 5A,D), three distinct phenotypic characteristics were evident in D. citri after silencing of the DcTPS gene. Firstly, fifth-instar nymphs were not able to complete a normal molt before death (Figure 5E). Secondly, some of the emerged adults fed with dsDcTPS showed "misshapen wings" (Figure 5B,F). Thirdly, partially deformed adults could not get rid of the cuticle from the nymphal stage (Figure 5C). Individuals from the dsGFP control group did not exhibit prominent variation in phenotype.

\subsection{Analysis of Trehalose-6-Phosphate Synthase, Trehalose and Glucose Contents}

Compared with dsGFP, the content of trehalose-6-phosphate synthase in $D$. citri was prominently lower at $24 \mathrm{~h}\left(\mathrm{~F}_{3,8}=60.087, p=0.0001\right)$ and $48 \mathrm{~h}\left(\mathrm{~F}_{3,8}=66.218, p=0.0001\right)$ (Figure. $\left.7 \mathrm{~A}\right)$. At the same time, the content of trehalose was also prominently lower at $24 \mathrm{~h}\left(\mathrm{~F}_{3,8}=128.727, p=0.0001\right)$ and $48 \mathrm{~h}\left(\mathrm{~F}_{3,8}=113.991, p=0.0001\right)$ (Figure $\left.7 \mathrm{~B}\right)$. However, the content of glucose was noticeably higher (Figure 7C) $24 \mathrm{~h}\left(\mathrm{~F}_{3,8}=98.052, p=0.0001\right)$ and $48 \mathrm{~h}\left(\mathrm{~F}_{3,8}=464.652, p=0.0001\right)$ after being fed with dsDcTPS. The results demonstrate the success of RNAi and indicate that downregulation of DcTPS has a considerable influence on DcTPS activity and the synthesis of trehalose in D. citri.
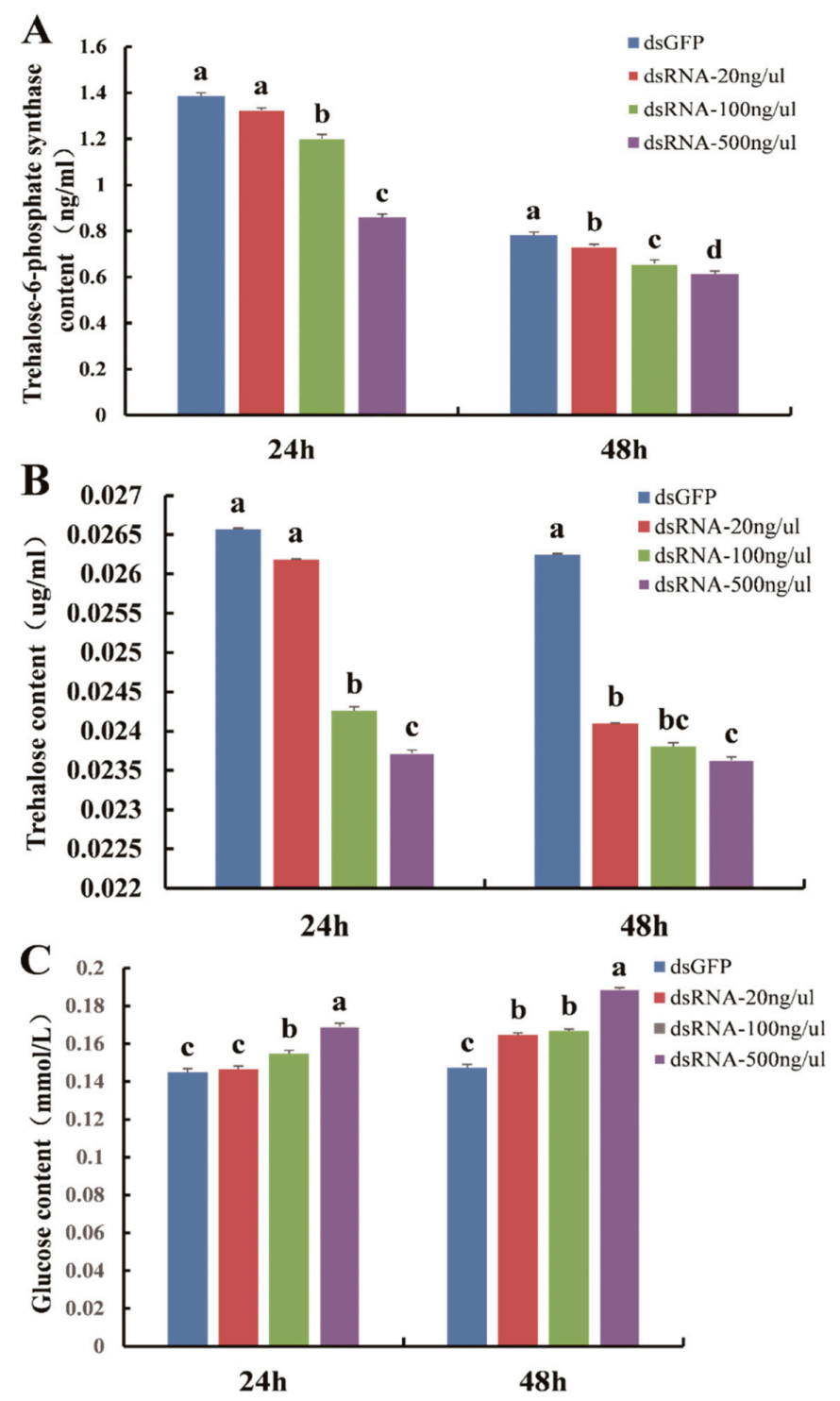

Figure 7. Detection of trehalose-6-phosphate synthase content (A), trehalose content (B) and glucose content (C) in D. citri after feeding dsRNA for 24 and $48 \mathrm{~h}$. Bars with different letters are significantly different according to SNK test $(p<0.05)$. 


\section{Discussion}

In this study, a cDNA sequence encoding TPS from D. citri was cloned and characterized for the first time. Sequence analysis showed that there are HDYHL and DGMNLV motifs in the putative DCTPS amino acid sequence. This result is consistent with previous studies [20,34]. Furthermore, multiple sequence alignment and the phylogenetic tree demonstrate higher identity and a closer evolutionary relationship of DcTPS with TPS from other Hemipteran insects such as A. pisum, D. noxia and N. lugens. These results are in accordance with the fact that TPS genes of similar species might have a closer evolutionary relationship and therefore could be clustered together $[3,21,34]$.

The expression of the DCTPS gene in multiple developmental stages has been reported in S. exigua [34], N. lugens [21], L. decemlineata [39], H. armigera [40] and M. domestica [41]. The result indicates that it might have a unique role in insect growth and development [20]. Like other insects, the expression level of the DCTPS gene was found to be stage specific. Its expression was highest before molting, which has been verified in many insect species $[20,23,41]$. Therefore, DcTPS may be related to molting, and this is possibly related to the requirement for trehalose during metamorphosis. Trehalose is a stored energy source for flight and can be hydrolyzed by cleavage of the glycosidic linkage to release two molecules of glucose and release of substantial energy to meet demand [26,42]. As a trehalose synthase, energy requirements of adults for flight should result in higher expression of DcTPS at this stage [41]. On the other hand, higher expression in adults may be also related to energy requirements for mating and spawning [26].

Many previous research reports have demonstrated the feasibility of RNAi through feeding $[8,11,21,30]$. Our results have shown that feeding-based RNAi of the DcTPS gene can specifically restrain the expression of the DCTPS gene and significantly affects nymphal growth and development in D. citri, leading to increased mortality and deformity. Consistent with these studies, RNAi of BmTPS and HvTPS decreased the larval survival rate and caused mortality or deformed phenotypes in B. minax [20] and H. vitessoides [23]. Many adults of D. citri did not molt normally and presented with misshapen wings after molting as a result of feeding on dsDcTPS. There might be many explanations for the molting abnormalities. Firstly, the regulation of chitin biosynthesis is related to the concentration of trehalose substrate and the expression of main genes in the pathway of chitin biosynthesis [21]. As a result of limited energy supply, chitin synthesis is affected when insects undergo metamorphosis [20,24,30,43]. Secondly, paucity of trehalose would weaken protection against abiotic stresses [23,29,32]. Thirdly, insect metamorphosis and wing formation are regulated by the TPS gene [21]. Meanwhile, lack of trehalose might weaken energy metabolism in healthy individuals of D. citri $[21,41]$. Therefore, there are some negative effects on the growth of nymphs and adults which cause even higher mortality and malformation in D. citri following the knockdown of the DcTPS gene. These results suggest that RNAi technology could be used for in-field control of D. citri [8].

Knockdown of the DCTPS gene significantly decreased DcTPS and trehalose content in D. citri. TPS is involved in trehalose synthesis as an important enzyme [26]. Silencing of the DcTPS gene could further weaken trehalose synthesis and lessen the trehalose content in D. citri. Consequently, a sharp drop in trehalose content could make nymphs more susceptible to stress conditions [41]. Trehalose is not only an energy store but also plays a crucial role in combating the negative effects of stress [26]. Moreover, the content of glucose increased in D. citri after RNAi. The reason behind this result may be that less reactants are involved in the trehalose synthesis chain so that glucose is continually accumulated. Our results are also in keeping with previous research showing that there is a negative correlation between trehalose and glucose content in insects [34].

\section{Conclusions}

Full-length cDNA of the DcTPS gene in D. citri was cloned for the first time. Developmental stage expression analysis showed that DcTPS expression was the highest at the fifth-instar nymph stage. In addition, there is a sharp drop in the expression of DcTPS and the number of surviving D. citri individuals attributed to dsRNA-mediated gene-specific silencing. There is also clear evidence of 
enhanced malformation. The significant change in trehalose and glucose concentration after RNAi suggests that DCTPS is related to trehalose metabolism of D. citri. These results establish a foundation for future studies on the physiological function of the DcTPS gene and provide a potential pest control target for management of $D$. citri. In our next study, we will design some novel biological insecticides which are directly targeted at $D$. citri trehalose metabolism and combined with RNAi to control D. citri in the field.

Author Contributions: Conceptualization: X.L. (Xinyu Liu), B.X.; Data curation: X.L. (Xinyu Liu), C.Z.; Investigation: X.L. (Xian Liu); Methodology: X.L. (Xinyu Liu), Z.Z., X.L. (Xian Liu), B.X.; Software: X.L. (Xinyu Liu); Writing-original draft: X.L. (Xinyu Liu); Writing-review \& editing: J.W., T.X., B.X. All authors have read and agreed to the published version of the manuscript.

Funding: This research was funded by the Leadership in Major Subjects Project of Jiangxi Province (20172BCB22004) National Natural Science Foundation of China (31860601 and 31760621), Jiangxi Key Research \& Development Plan, China (20161BBF60117), Science and Technology Project Founded by the Education Department of Jiangxi Province (GJJ14167).

Conflicts of Interest: The authors declare no conflict of interest.

\section{References}

1. Liu, B.; Coy, M.R.; Wang, J.J.; Stelinski, L.L. Characterization of the voltage-gated sodium channel of the Asian citrus psyllid, Diaphorina citri. Insect Sci. 2016, 24, 47-59. [CrossRef] [PubMed]

2. Liu, X.Q.; Jiang, H.B.; Xiong, Y.; Peng, P.; Li, H.F.; Yuan, G.R.; Dou, W.; Wang, J.J. Genome-wide identification of ATP-binding cassette transporters and expression profiles in the Asian citrus psyllid, Diaphorina citri, exposed to imidacloprid. Comp. Biochem. Physiol. Part D 2019, 30, 305-311. [CrossRef] [PubMed]

3. Santos-Ortega, Y.; Killiny, N. Silencing of sucrose hydrolase causes nymph mortality and disturbs adult osmotic homeostasis in Diaphorina citri (Hemiptera: Liviidae). Insect Biochem. Mol. Biol. 2018, 101, 131-143. [CrossRef] [PubMed]

4. Chen, X.D.; Ashfaq, M.; Stelinski, L.L. Susceptible of Asian citrus psyllid, Diaphorina citri (Hemiptera: Liviidae), to the insecticide afidopyropen: A new and potent modulator of insect transient receptor potential channels. Appl. Entomol. Zoolog. 2018, 53, 453-461. [CrossRef]

5. Qureshi, J.A.; Kostyk, B.C.; Stansly, P.A. Insecticidal suppression of Asian citrus psyllid Diaphorina citri (Hemiptera: Liviidae) vector of huanglongbing pathogens. PLoS ONE 2014, 9, e112331. [CrossRef]

6. Xiong, X.; Wu, L.Y.; Xin, T.R.; Wang, J.; Zou, Z.W.; Xia, B. The complete mitochondrial genome of Diaphorina citri (Hemiptera: Psyllidae) and phylogenetic analysis. Biochem. Syst. Ecol. 2017, 70, 230-238. [CrossRef]

7. Zhang, C.; Xiong, X.; Liu, X.; Zou, Z.W.; Xin, T.R.; Wang, J.; Xia, B. Diaphorina citri (Hemiptera: Psylloidea) in China: Two Invasion Routes and Three Transmission Paths. J. Econ. Entomol. 2019, 112, 1418-1427. [CrossRef]

8. Lu, Z.J; Huang, Y.L.; Yu, H.Z.; Li, N.Y.; Xie, Y.X.; Zhang, Q.; Zeng, X.D.; Hu, H.; Huang, A.J.; Yi, L.; et al. Silencing of the Chitin Synthase Gene Is Lethal to the Asian Citrus Psyllid, Diaphorina citri. Int. J. Mol. Sci. 2019, 20, 3734. [CrossRef]

9. Boina, D.R.; Bloomquist, J.R. Chemical control of the Asian citrus psyllid and of huanglongbing disease in citrus. Pest Manag. Sci. 2015, 71, 808-823. [CrossRef]

10. Kruse, A.; Fattah-Hosseini, S.; Saha, S.; Johnson, R.; Warwick, E.R.; Sturgeon, K.; Mueller, L.; Maccoss, M.J.; Shatters, R.G.; Heck, M.C. Combining' omics and microscopy to visualize interactions between the Asian citrus psyllid vector and the Huanglongbing pathogen Candidatus Liberibacter asiaticus in the insect gut. PLoS ONE 2017, 12, e0179531. [CrossRef]

11. Yu, H.Z.; Huang, Y.L.; Lu, Z.J.; Zhang, Q.; Su, H.N.; Du, Y.M.; Yi, L.; Zhong, B.L.; Chen, C.X. Inhibition of trehalase affects the trehalose and chitin metabolism pathways in Diaphorina citri (Hemiptera: Psyllidae). Insect Sci. 2020. [CrossRef] [PubMed]

12. Huang, J.K.; Hu, R.F.; Pray, C.; Qiao, F.B.; Rozelle, S. Biotechnology as an alternative to chemical pesticides: A case study of Bt cotton in China. J. Agric. Econ. 2003, 29, 55-67. [CrossRef]

13. Tiwari, S.; Clayson, P.J.; Kuhns, E.H.; Stelinski, L.L. Effects of buprofezin and diflubenzuron on various developmental stages of Asian citrus psyllid, Diaphorina citri. Pest Manag. Sci. 2012, 68, 1405-1412. [CrossRef] [PubMed] 
14. Eaton, B.A.; Fetter, R.D.; Davis, G.W. Dynactin is necessary for synapse stabilization. Neuron 2002, 34, 729-741. [CrossRef]

15. Mao, Y.B.; Cai, W.J.; Wang, J.W.; Hong, G.J.; Tao, X.Y.; Wang, L.J.; Huang, Y.P.; Chen, X.Y. Silencing a cotton bollworm P450 monooxygenase gene by plant-mediated RNAi impairs larval tolerance of gossypol. Nat. Biotech. 2007, 25, 1307-1313. [CrossRef] [PubMed]

16. Chen, X.F.; Tian, H.G.; Zou, L.; Tang, B.; Hu, J.; Zhang, W.Q. Disruption of Spodoptera exigua larval development by silencing chitin synthase gene A with RNA interference. Bull. Entomol. Res. 2008, 98, 613-619. [CrossRef] [PubMed]

17. Dzitoyeva, S.; Dimitrijevic, N.; Manev, H. Intra-abdominal injection of double-stranded RNA into anesthetized adult Drosophila triggers RNA interference in the central nervous system. Mol. Psychiatr. 2001, 6, 665-670. [CrossRef]

18. Tomoyasu, Y.; Miller, S.C.; Tomita, S.; Schoppmeier, M.; Grossmann, D.; Bucher, G. Exploring systemic RNA interference in insects: A genome-wide survey for RNAi genes in Tribolium. Genome Biol. 2008, 9, R10. [CrossRef]

19. Bhatia, V.; Bhattacharya, R. Host-mediated RNA interference targeting a cuticular protein gene impaired fecundity in the green peach aphid Myzus persicae. Pest Manag. Sci. 2018, 74, 2059-2068. [CrossRef]

20. Chen, J.X.; Lyu, Z.H.; Wang, C.Y.; Cheng, J.; Lin, T. RNA interference of a trehalose-6-phosphate synthase gene reveals its roles in the biosynthesis of chitin and lipids in Heortia vitessoides (Lepidoptera: Crambidae). Insect Sci. 2018, 0, 1-12. [CrossRef]

21. Chen, J.; Zhang, D.; Yao, Q.; Zhang, J.; Dong, X.; Tian, H.; Chen, J.; Zhang, W. Feeding-based RNA interference of a trehalose phosphate synthase gene in the brown planthopper, Nilaparvata lugens. Insect Mol. Biol. 2010, 19, 777-786. [CrossRef] [PubMed]

22. Chen, Q.W.; Jin, S.; Zhang, L.; Shen, Q.D.; Wei, P.; Wei, Z.M.; Wang, S.G.; Tang, B. Regulatory functions of trehalose-6-phosphate synthase in the chitin biosynthesis pathway in Tribolium castaneum (Coleoptera: Tenebrionidae) revealed by RNA interference. Bull. Entomol. Res. 2017, 108, 388-399. [CrossRef] [PubMed]

23. Xiong, K.C.; Wang, J.; Li, J.H.; Deng, Y.Q.; Pu, P.; Fan, H.; Liu, Y.H. RNA interference of a trehalose-6-phosphate synthase gene reveals its roles during larval-pupal metamorphosis in Bactrocera minax (Diptera: Tephritidae). J. Insect Physiol. 2016, 91-92, 84-92. [CrossRef] [PubMed]

24. Yang, M.M.; Zhao, L.N.; Shen, Q.D.; Xie, G.Q.; Wang, S.G.; Tang, B. Knockdown of two trehalose-6-phosphate synthases severely affects chitin metabolism gene expression in the brown planthopper Nilaparvata lugens. Pest Manag. Sci. 2016, 73, 206-216. [CrossRef]

25. Becker, A.; Schlöder, P.; Steele, J.E.; Wegener, G. The regulation of trehalose metabolism in insects. Experientia 1996, 52, 433-439. [CrossRef]

26. Elbein, A.D.; Pan, Y.T.; Pastuszak, I.; Carroll, D. New insights on trehalose: A multifunctional molecule. Glycobiology 2003, 13, 17R-27R. [CrossRef]

27. Chen, Q. Role of trehalose phosphate synthase and trehalose during hypoxia: From flies to mammals. J. Exp. Biol. 2004, 207, 3125-3129. [CrossRef]

28. Tang, B.; Chen, X.; Liu, Y.; Tian, H.; Liu, J.; Hu, J. Characterization and expression patterns of a membranebound trehalase from Spodoptera exigua. BMC Mol. Biol. 2008, 9, 51. [CrossRef]

29. Wyatt, G.R. The biochemistry of sugars and polysaccharides in insects. Adv. Insect Physiol. 1967, 4, $287-360$. [CrossRef]

30. Tang, B.; Wei, P.; Zhao, L.N.; Shi, Z.K.; Shen, Q.D.; Yang, M.M.; Xie, G.Q.; Wang, S.G. Knockdown of five trehalase genes using RNA interference regulates the gene expression of the chitin biosynthesis pathway in Tribolium castaneum. BMC Biotechnol. 2016, 16, 67. [CrossRef]

31. Tang, B.; Wang, S.; Wang, S.G.; Wang, H.J.; Zhang, J.Y.; Cui, S.Y. Invertebrate Trehalose-6-Phosphate Synthase Gene: Genetic Architecture, Biochemistry, Physiological Function, and Potential Applications. Front. Physiol. 2018, 9. [CrossRef] [PubMed]

32. Chen, Q.; Ma, E.; Behar, K.L.; Xu, T.; Haddad, G.G. Role of trehalose phosphate synthase in anoxia tolerance and development in Drosophila melanogaster. J. Biol. Chem. 2002, 277, 3274-3279. [CrossRef] [PubMed]

33. Cui, S.Y.; Xia, Y.X. Isolation and characterization of the trehalose-6-phosphate synthase gene from Locusta migratoria manilensis. Insect Sci. 2009, 16, 287-295. [CrossRef] 
34. Tang, B.; Chen, J.; Yao, Q.; Pan, Z.Q.; Xu, W.H.; Wang, S.G. Characterization of a trehalose-6-phosphate synthase gene from Spodoptera exigua, and its function identification through RNA interference. J. Insect Physiol. 2010, 56, 813-821. [CrossRef]

35. Tang, B.; Zheng, H.Z.; Xu, Q.; Zou, Q.; Wang, G.J.; Zhang, F.; Wang, S.G.; Zhang, Z.H. Cloning and pattern of expression of trehalose-6-phosphate synthase cDNA from Catantops pinguis (Orthoptera: Catantopidae). Eur. J. Entomol. 2011, 108, 355-363. [CrossRef]

36. Tsai, J.H.; Liu, Y.H. Biology of Diaphorina citri (Homoptera: Psyllidae) on four host plants. J. Econ. Entomol. 2000, 93, 1721-1725. [CrossRef]

37. Kumar, S.; Stecher, G.; Tamura, K. MEGA7: Molecular evolutionary genetics analysis version 7.0 for bigger datasets. Mol. Biol. Evol. 2016, 33, 1870-1874. [CrossRef]

38. Livak, K.J.; Schmittgen, T.D. Analysis of relative gene expression data using real-time quantitative PCR and the $2^{-\Delta \Delta C T}$ method. Methods Enzymol. 2001, 25, 402-408. [CrossRef]

39. Shi, J.F.; Xu, Q.Y.; Guo, W.C.; Li, G.Q. Transcription changes of a putative trehalose-6-phosphate synthase gene in response to hormone stimulation in Leptinotarsa decemlineata, (Say). J. Asia-Pac. Entomol. 2016, 19, 775-783. [CrossRef]

40. Xu, J.; Bao, B.; Zhang, Z.F.; Yi, Y.Z.; Xu, W.H. Identification of a novel gene encoding the trehalose phosphate synthase in the cotton bollworm, Helicoverpa armigera. Glycobiology 2009, 19, 250-257. [CrossRef]

41. Zhang, Y.; Wang, F.; Feng, Q.; Wang, H.; Tang, T.; Huang, D.; Liu, F. Involvement of trehalose-6-phosphate synthase in innate immunity of Musca domestica. Dev. Comp. Immunol. 2019, 91, 85-92. [CrossRef] [PubMed]

42. Sola-Penna, M.; Meyer-Fernandes, J.R. Stabilization against Thermal Inactivation Promoted by Sugars on Enzyme Structure and Function: Why Is Trehalose More Effective than Other Sugars? Arch. Biochem. Biophys. 1998, 360, 10-14. [CrossRef] [PubMed]

43. Shukla, E.; Thorat, L.J.; Nath, B.B.; Gaikwad, S.M. Insect trehalase: Physiological significance and potential applications. Glycobiology 2018, 25, 357-367. [CrossRef] [PubMed]

(C) 2020 by the authors. Licensee MDPI, Basel, Switzerland. This article is an open access article distributed under the terms and conditions of the Creative Commons Attribution (CC BY) license (http://creativecommons.org/licenses/by/4.0/). 